\title{
Nemo-like kinase expression predicts poor survival in colorectal cancer
}

\author{
JINGBO CHEN $^{1 *}$, YUNWEI HAN ${ }^{2,3 *}$, XIAOQIAN ZHAO ${ }^{4}$, MINGYU YANG $^{1}$, BO LIU $^{1}$, XIANGPENG XI $^{1}$, \\ XIAOLIN XU ${ }^{1}$, TIEJUN LIANG ${ }^{4}$ and LIJIAN XIA ${ }^{1}$ \\ ${ }^{1}$ Department of Gastrointestinal Surgery, Qianfoshan Hospital of Shandong Province; ${ }^{2}$ School of Medicine, \\ Shandong University, Jinan, Shandong 250100; ${ }^{3}$ Department of Oncology, Affiliated Hospital of \\ Luzhou Medical College, Luzhou, Sichuan 646000; ${ }^{4}$ Department of Digestive Diseases, Shandong \\ Provincial Hospital Affiliated to Shandong University, Jinan, Shandong 250014, P.R. China
}

Received December 18, 2013; Accepted September 12, 2014

DOI: $10.3892 / \mathrm{mmr} .2014 .2851$

\begin{abstract}
Nemo-like kinase (NLK), a serine/threonine protein kinase, was previously reported to be associated with tumor proliferation and invasion. The present study aimed to evaluate whether NLK participates in the tumorigenesis and progression of colorectal cancer (CRC). NLK expression was examined using reverse transcription quantitative polymerase chain reaction (RT-qPCR) and western blot analysis in 50 paired CRC tissues as well as immunohistochemical analysis of 406 cases of primary CRC tissues and paired non-cancerous tissues. Correlations between NLK expression, the clinicopathological features of CRC patients and clinical outcome were then analyzed. NLK expression was found to be significantly higher in CRC tissues as well as associated with the depth of tumor invasion, lymph node metastasis, distant metastasis, histological differentiation, vascular invasion and advanced tumor stage. Patients with NLK-positive tumors demonstrated higher rates of recurrence and mortality than patients with NLK-negative tumors. Multivariate analyses revealed that NLK expression was an independent factor for overall survival [hazard ratio $(\mathrm{HR})=0.035$; $95 \%$ confidence interval $(\mathrm{CI})=0.02-0.19 ; \mathrm{P}<0.001]$ and disease-free survival $(\mathrm{HR}=0.033 ; 95 \% \mathrm{CI}=0.007-0.09 ; \mathrm{P}<0.001)$ in $\mathrm{CRC}$ patients. In conclusion, the results of the present study indicated that NLK may serve as a novel biomarker for tumor recurrence and survival for CRC patients.
\end{abstract}

Correspondence to: Dr Lijian Xia, Department of Gastrointestinal Surgery, Qianfoshan Hospital of Shandong Province, 16766 of 10 Run Road, Jinan, Shandong 250014, P.R. China

E-mail: xiaalbert@126.com

*Contributed equally

Key words: colorectal cancer, nemo-like kinase, prognosis, recurrence, biomarker

\section{Introduction}

Colorectal cancer (CRC) is a prevalent type of cancer, which has a high mortality rate worldwide (1). In Europe and the USA, CRC is the third most common type of human cancer and the second leading cause of cancer-associated mortality (2,3). In China, the incidence of $\mathrm{CRC}$ has risen steadily over the last few decades, with increasing morbidities in younger patients ( $<50$ years) (4). Recent cancer statistics have indicated that CRC accounts for $\sim 9 \%$ of all cancer-associated mortalities (5). The survival rate of CRC is higher with earlier diagnoses followed by treatment with surgical resection; however, the long-term survival and prognosis of the patients at stages III and IV remain poor (6). Genes associated with mutations in $\operatorname{TP} 53(7), \operatorname{KRAS}(8,9)$ and $B R A F(9,10)$ as well as defective DNA mismatch repair (11) have been investigated for their prognostic and predictive value in CRC; however, the application of these markers requires validation in clinical practice and further evaluation. Sensitive biomarkers enable an early diagnosis and prognosis prediction; therefore, novel factors for predicting tumor recurrence and prognosis following surgery are urgently required.

The Wnt signaling pathway and its downstream components have a role in the regulation of cancer progression through numerous processes, including tumor initiation, tumor growth, cell senescence, cell death, differentiation and metastasis (12). The Wnt signaling pathway molecule Nemo-like kinase (NLK) is a member of the extracellular-signal regulated kinase/mitogen-activated protein kinase (MAPK) and cyclin-dependent kinase families (13). NLK was reported to induce apoptosis and inhibit androgen receptor-mediated transcriptional activity in prostate cancer cells (14). However, NLK also contributes to tumor growth via the activation of cell cycle proteins cyclin D1 and cyclin-dependent kinase 2 in human hepatocellular carcinoma (15). NLK was also demonstrated to induce apoptosis in glioma cells via activation of caspases (16). These previous studies have indicated that NLK may be a critical regulator of tumor growth and development. In the present study, reverse transcription quantitative polymerase chain reaction (RT-qPCR) and immunohistochemical 
analysis were used to determine whether there was an association between NLK expression and the clinical outcome of CRC patients.

\section{Materials and methods}

Tissue specimens and patient information. A total of 406 clinical specimens were collected from the medical records of patients with CRC who underwent surgery at the Department of Gastrointestinal Surgery of Qianfoshan Hospital of Shandong Province and the Department of Digestive Diseases of Shandong Provincial Hospital Affiliated to Shandong University (Shandong, China). All specimens were archived under protocols approved by the institutional review boards of Shandong University and written informed consent was obtained from the patients. The group was composed of 172 males and 234 females with a mean \pm standard error of the mean age of $64.8 \pm 17.1$ (range, 23-91) years. The diagnoses were confirmed by two pathologists and based on the tumor, node, metastasis classification system: 48 cases at stage I, 162 cases at stage II, 160 cases at stage III and 36 cases at stage IV. Among these patients, 132 had lymph node metastases (LNM). The follow-up of CRC patients post-surgery was performed according to the National Comprehensive Cancer Network Practice guidelines. Overall survival (OS) and disease-free survival (DFS) rates were defined as the interval from the initial surgery to clinically or radiologically proven recurrence/metastasis and mortality, respectively.

The patients were enrolled in the present study between 2006 and 2009. The follow-up for all cases was terminated in February 2012. During survival analysis, cases were regarded as censored data when patients were lost to follow-up or succumbed to their disease.

$R N A$ extraction and $R T-q P C R$. Total RNA extraction of 50 paired freshly frozen primary tumor and adjacent normal mucosa (10 cm away from the original tumor site) of CRC specimens were performed according to the manufacturer's instructions (Qiagen, Shanghai, China). A Reverse Transcription kit (Qiagen) was used to reverse transcribe total RNA according to the manufacturer's instructions. Quantitative PCR was performed using a SYBR Green PCR kit (Thermo Scientific, Waltham, MA, USA) according to the manufacturer's instructions. The human NLK gene was amplified using a commercial NLK qPCR Primer Pair (NM_016231; OriGene, Rockville, MD, USA) and $\beta$-actin (HP204660; OriGene) was used as the internal control. Cycling conditions were as follows: Denaturation $\left(5 \mathrm{~min}\right.$ at $\left.93^{\circ} \mathrm{C}\right)$ followed by 40 cycles of denaturation $\left(30 \mathrm{sec}\right.$ at $\left.93^{\circ} \mathrm{C}\right)$, annealing $\left(15 \mathrm{sec}\right.$ at $\left.58^{\circ} \mathrm{C}\right)$ and elongation $\left(1 \mathrm{~min}\right.$ at $\left.72^{\circ} \mathrm{C}\right)$. Each reaction was performed in triplicate and the $2^{-\Delta \Delta \mathrm{Ct}}$ method was used to calculate relative expression.

Western blot analysis. Western blot analysis was performed as previously described (17). Monoclonal human anti-NLK antibodies (1:1,000; Cell Signaling Technology, Inc., Danvers, MA, USA) and monoclonal anti- $\beta$-actin antibodies $(1: 2,000$; Beyotime Biotechnology, Jiangsu, China) were used as primary antibodies. Immunoreactive bands were detected using a Phototope-horseradish peroxidase western blot detection

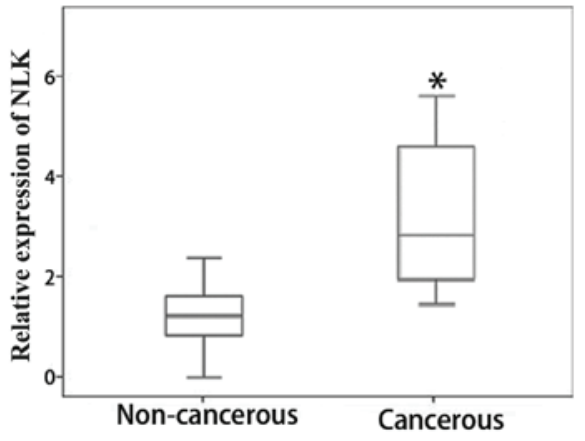

Figure 1. Reverse transcription polymerase chain reaction was performed in order to quantify the differences in nemo-like kinase expression between cancerous and adjacent non-cancerous control tissues $(n=50)$. ${ }^{*} \mathrm{P}<0.01$ vs. non-cancerous tissue. $\mathrm{Ct}$, cycle threshold. Boxes represent percentiles. Bars within represents the median. Error bars represent standard error

kit (Cell Signaling Technology, Inc.). For densitometric analysis, NLK protein bands on the blots were measured using Image J software (National Institutes of Health, Bethesda, MD, USA) following normalization to the corresponding $\beta$-actin expression levels.

Immunohistochemical analysis. Paraffin-embedded sections fixed in formalin were deparaffinized, rehydrated and incubated with 3\% hydrogen peroxidase (Qiagen). The sections were then heated in a microwave oven $(1,000$ Watts 8503 ; Kenmore, Chicago, IL, USA) for $3 \mathrm{~min}$ at $100^{\circ} \mathrm{C}$ for antigen retrieval. Slides were incubated with blocking serum (Qiagen) and primary antibodies for NLK (1:100) overnight at $4^{\circ} \mathrm{C}$. The immunohistochemical reaction was visualized using $0.05 \%$ diaminobenzidine followed by counterstaining with hematoxylin. Sections were then examined and analyzed using a microscope (Leica M80; Leica Microsystems, Wetzlar, Germany). Negative control sections were incubated with preimmune rabbit serum (Qiagen) instead of the primary antibodies.

Immunostaining was defined independently using two pathologists blinded to the clinical data and scored by multiplying the staining intensity and the percentage of the stained tumor cells. Staining intensity was graded from 0-3 and the percentage of the stained tumor cells was graded as follows: $0,<5 \% ; 1,5-25 \% ; 2,26-50 \% ; 3,51-75 \%$; and 4 , $>75 \%$. Final scores ranged from 0 to 12 . Samples with overall scores from 0-4 were defined as negative expression, while the samples with scores 5-12 were grouped and defined as positive expression (18). Specimens with inconsistent scores were re-evaluated by two pathologists until an agreement was reached.

Statistical analysis. For categorical variables, values are expressed as the numerical count and the $\chi^{2}$ test or Fisher's exact test were used to determine the statistical significance of differences between NLK and clinicopathological variables. Kaplan-Meier curves with log-rank tests represented the cumulative survival rate for OS and DFS using NLK expression levels. The Cox proportional hazards model was used to calculate univariate and multivariate hazard ratios 
Table I. NLK expression in adjacent normal mucosa, cancerous tissues and LNM tissues.

\begin{tabular}{|c|c|c|c|c|}
\hline \multirow[b]{2}{*}{ Tissue sample } & \multirow[b]{2}{*}{$\mathrm{n}$} & \multicolumn{2}{|c|}{ Expression of NLK } & \multirow[b]{2}{*}{ P-value } \\
\hline & & Negative, $\mathrm{n}(\%)$ & Positive, $\mathrm{n}(\%)$ & \\
\hline Normal mucosa & 406 & 364 (89.7) & $42(9.9)$ & $<0.001^{\mathrm{a}}$ \\
\hline Cancerous & 406 & $172(42.4)$ & $234(57.6)$ & $<0.001^{\mathrm{a}}$ \\
\hline LNM & 132 & $12(9.1)$ & $120(90.9)$ & $<0.001^{\mathrm{a}}$ \\
\hline
\end{tabular}

${ }^{a} \mathrm{P}<0.01$ vs. negative NLK expression. NLK, nemo-like kinase; LNM, lymph node metastasis.

Table II. NLK expression and clinicopathological characteristics in colorectal cancer.

\begin{tabular}{lrrr}
\hline & \multicolumn{2}{l}{ NLK protein expression } & \\
\cline { 2 - 3 } Variable & $\begin{array}{c}\text { Negative } \\
(\mathrm{n}=172)\end{array}$ & $\begin{array}{c}\text { Positive } \\
(\mathrm{n}=234)\end{array}$ & P-value \\
\hline Age & & & \\
$<65$ & 76 & 86 & 0.586 \\
$\geq 65$ & 96 & 148 & \\
Gender & & & \\
Male & 72 & 100 & 0.374 \\
Female & 100 & 134 & \\
pT stage & & & \\
pT1 & 8 & 8 & $<0.001^{\mathrm{a}}$ \\
pT2 & 34 & 12 & \\
pT3 & 72 & 80 & \\
pT4 & 58 & 134 & \\
pN stage & & & \\
pN0 & 166 & 50 & $0.001^{\mathrm{a}}$ \\
pN1 & 2 & 120 & \\
pN2 & 4 & 64 & \\
M stage & & & \\
M0 & 170 & 50 & $<0.001^{\mathrm{a}}$ \\
M1 & 2 & 15 & \\
Vessel invasion & & & \\
No & 168 & 200 & $0.018^{\mathrm{a}}$ \\
Yes & 4 & 34 & \\
Differentiation & & & \\
Good & 102 & 96 & $0.0015^{\mathrm{a}}$ \\
Moderate/poor & 70 & 140 & \\
\hline
\end{tabular}

${ }^{\text {aP }}<0.01$ vs. negative NLK expression. NLK, nemo-like kinase; $\mathrm{pT}$, tumor invasion depth; $\mathrm{pN}$, lymph node metastasis; M, distant metastasis.

for the study variables. $\mathrm{P}<0.01$ was considered to indicate a statistically significant difference between values. All statistical analyses were performed using the SPSS 17.0 statistical software package (SPSS, Inc., Chicago, IL, USA).
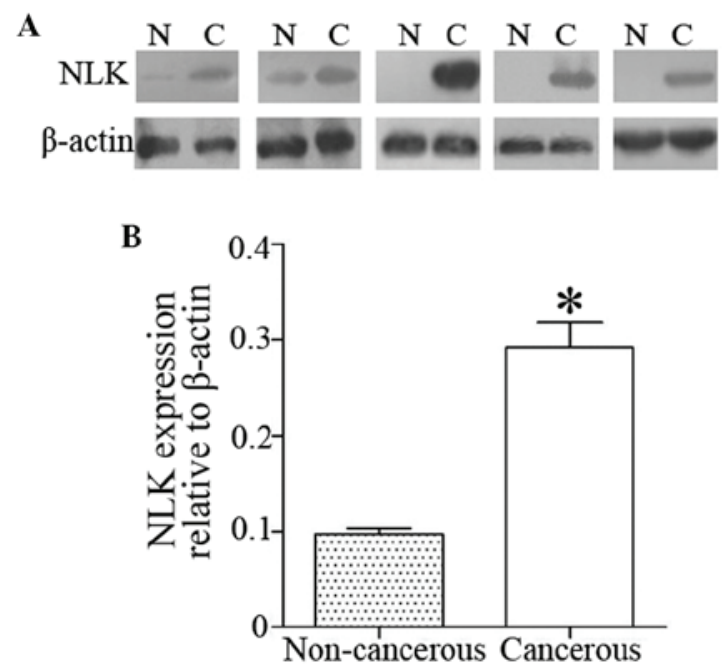

Figure 2. Western blot analysis of NLK in cancerous and adjacent non-cancerous tissues. (A) Representative western blots and (B) relative expression of NLK levels in cancerous and adjacent non-cancerous paired colorectal tissues of patients. $\beta$-actin was used as internal control and the $\chi^{2}$ test was used for statistical analyses. " $\mathrm{P}<0.01$ vs. non-cancerous tissue. NLK, nemo-like kinase; $\mathrm{N}$, non-cancerous; $\mathrm{C}$, cancerous.

\section{Results}

NLK upregulation in CRC tissues. Among the 50 paired specimens available for RT-qPCR analysis, the relative expression levels of NLK mRNA showed a minimum of a two-fold increase in $78.0 \%$ of tumor tissues compared to those of the adjacent normal mucosa (Fig. 1). This therefore suggested that NLK expression was upregulated in CRC tissues.

In addition, western blot analysis was used to confirm these results in the examined 50 paired tumors and corresponding normal tissues. The positive rate of NLK expression was $66.0 \%$ in CRC tissues and $18.0 \%$ in the matched non-cancerous normal tissues; therefore, NLK expression was significantly higher in CRC tissues than that in the matched normal colorectal tissues $(\mathrm{P}<0.01)$ (Fig. 2A and $\mathrm{B})$.

Correlation between NLK expression and clinicopathological features in CRC. In order to further analyze the clinical and pathological features of NLK expression, immunohistochemical analysis was used to detect NLK protein expression in 406 cases of CRC and paired adjacent noncancerous tissues (Fig. 3). The 

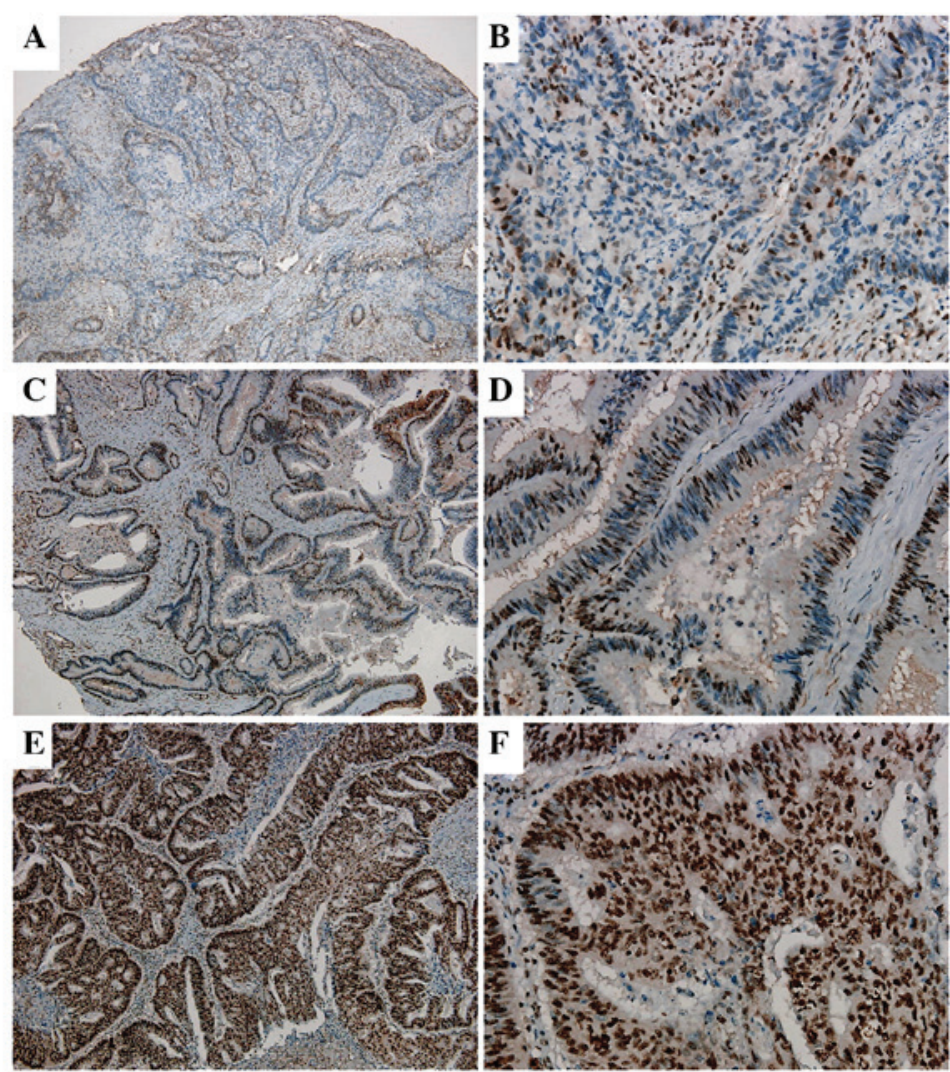

Figure 3. Immunohistochemical analysis of NLK expression in colorectal cancer and adjacent normal colorectal mucosa. (A and B) Weakly positive, 1+; (C and D) moderately positive, 2+; and (E and F) strongly positive, 3+ scoring for nuclear NLK staining [magnification, x100 (A, C, E) and $\mathrm{x} 400(\mathrm{~B}, \mathrm{D}, \mathrm{F})]$. NLK, nemo-like kinase.

A

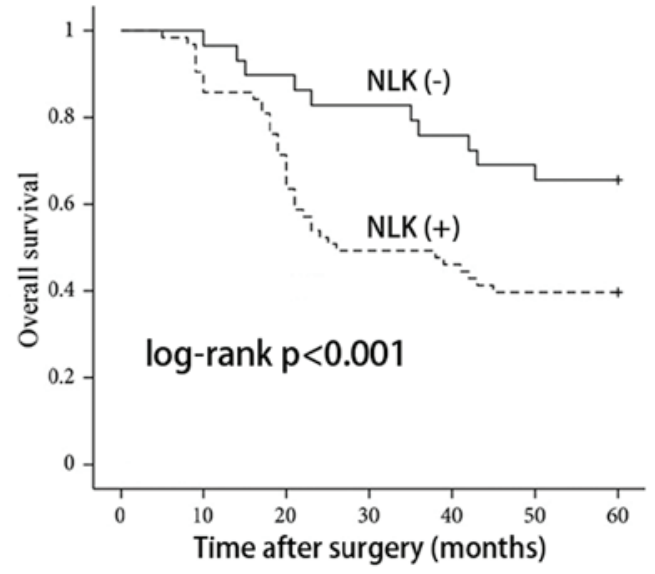

B

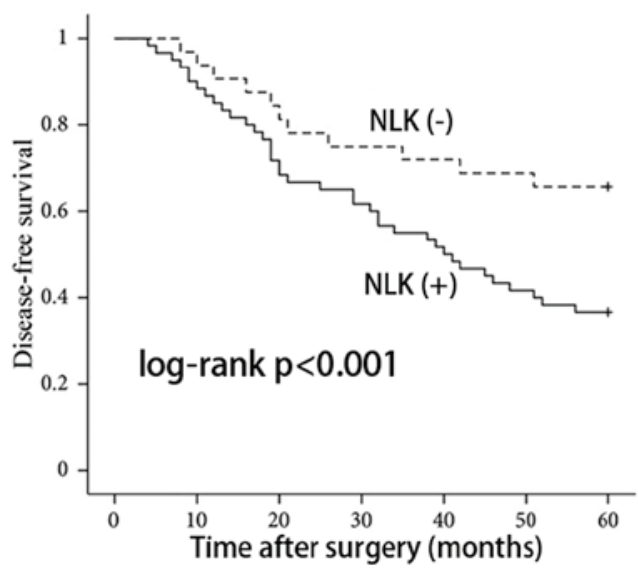

Figure 4. Kaplan-Meier analysis of (A) overall survival and (B) disease-free survival according to NLK expression levels following surgery. P<0.001 betwwn positive and negative NLK expression. NLK, nemo-like kinase.

results demonstrated that $89.7 \%$ of non-cancerous specimens were negative for NLK expression; by contrast, $57.6 \%$ of CRC specimens exhibited positive NLK expression. In addition, among the 132 LNM tissues, $90.9 \%$ displayed positive NLK expression (Table I).

The correlations between NLK protein expression and clinicopathological features are shown in Table II. The positive expression of NLK was significantly correlated with the depth of tumor invasion, LNM, distant metastasis, vascular invasion and histological differentiation. No significant correlations were observed between NLK expression and age or gender. NLK expression levels were found to be significantly higher in the nodal metastasis than those of the CRC and noncancerous tissues $(\mathrm{P}<0.001)$. These data indicated that increased NLK expression may correlate with CRC metastasis.

NLK expression and survival analysis. In order to assess the possible associations between NLK expression and 


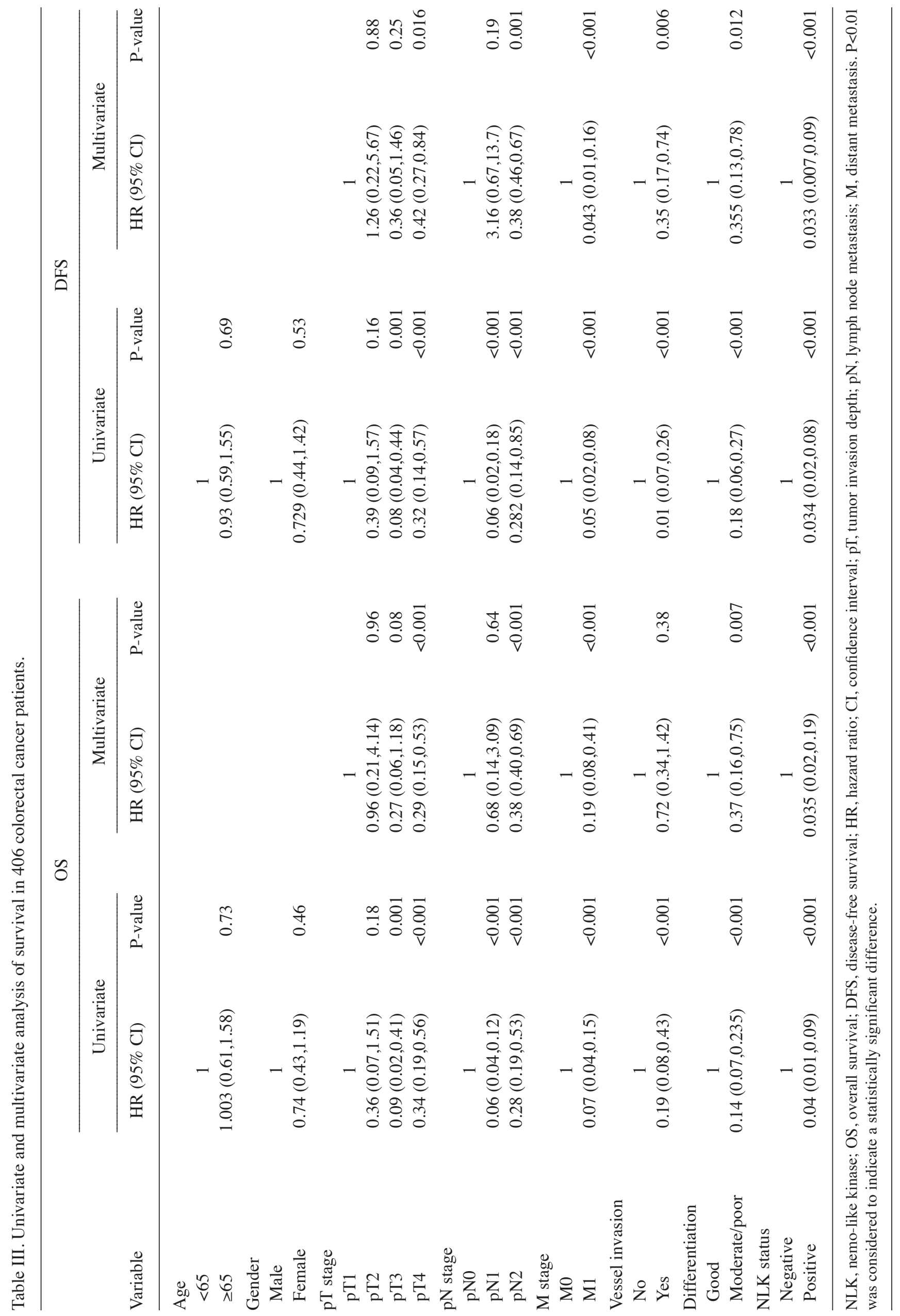


CRC patient survival, Kaplan-Meier curves using log-rank tests for OS and DFS were performed. As shown in Fig. 4, patients with positive NLK expression showed decreased rates of OS and DFS, respectively. In addition, patients with positive NLK expression had a higher recurrence rate than patients with negative expression $(\mathrm{P}<0.001$; data not shown). As shown in Table III, univariate analysis revealed that OS as well as DFS were significantly associated with advanced tumor stage, lymph node metastasis, distant metastasis, histological differentiation, vascular invasion and NLK expression. Multivariate analysis was performed using the Cox proportional hazards model, and the results demonstrated that positive NLK expression remained a significant independent prognostic factor for OS [hazard ratio $(\mathrm{HR})=0.035 ; 95 \%$ confidence interval $(\mathrm{CI})=0.02-0.19$; $\mathrm{P}<0.001]$ and DFS $(\mathrm{HR}=0.033 ; 95 \% \mathrm{CI}=0.007-0.09 ; \mathrm{P}<0.001)$.

\section{Discussion}

The results of the present study have demonstrated the correlation between elevated NLK expression and human CRC prognosis; of note, correlations were observed between positive NLK expression and aggressive features of CRC, including tumor depth, lymph node metastasis and distant metastasis. This therefore indicated the potential use of NLK as a tumor biomarker for CRC; furthermore, these results suggested that NLK may be used as a novel prognostic marker for more aggressive phenotypes of CRC patients following surgical resection.

The NLK gene, which encodes a proline-directed MAPK family member, was identified in 1994 (19). Functional analyses have demonstrated that NLK contributed to numerous signaling pathways via its ability to phosphorylate diverse transcription factors (20). NLK was reported to be a pivotal regulator of the $\mathrm{Wnt} / \beta$-catenin signaling pathway (21). In addition, NLK has also been shown to regulate the activity of multiple transcription factors, including NF- $\kappa \mathrm{B}$, Smads and p53 (22). Therefore, the reported involvement of NLK in numerous signaling pathways has demonstrated its vital role in mediating cell signals. Studies of NLK function in human cancers have also confirmed the role of NLK in cell growth and proliferation. In addition, NLK was reported to function as a tumor suppressor gene or an oncogene in different types of cancers; for example, data have demonstrated that NLK expression was decreased during prostate cancer progression and indicated that NLK inhibited androgen receptor (AR) expression and subsequent AR-mediated transcription as well as promoted apoptosis in prostate cancer cell lines (14). In a previous study, clinicopathological analysis revealed that NLK expression levels were significantly higher in human glioma tissues compared with those of lower grade tumors and the survival rate of glioma patients expressing low levels of NLK was significantly decreased compared to that of patients with gliomas expressing high levels of NLK (16). Conversely, NLK was reported to act as an oncogene in certain types of tumors. A previous study demonstrated the mitogenic potential of NLK in hepatocellular carcinomas through siRNA-mediated disruption of NLK, which was shown to inhibit proliferation of Hep3B cells and arrest cell cycle transition (15). These discrepancies may be due to the different pathologies of the types of tumors being studied. In the present study, NLK expression was shown to be significantly upregulated in CRC tissues compared to that of the paired non-cancerous samples, indicating the involvement of NLK in CRC progression.

A recent study reported that NLK overexpression was associated with the progression of gallbladder cancer and that NLK may have potential for use as a prognostic marker (23). Therefore, the present study aimed to analyze the correlations between NLK expression and the clinicopathological features of CRC patients as well as clinical outcome. These results revealed that positive NLK expression was significantly correlated with the depth of tumor invasion, lymph node metastasis, distant metastasis, histological differentiation, vascular invasion and advanced tumor stage. Kaplan-Meier survival analysis demonstrated that positive NLK expression was negatively correlated with the decreased overall survival rate of CRC patients. Of note, Cox multivariate analysis revealed that NLK expression was an independent factor in predicting OS and DFS for CRC patients. In conclusion, the results of the present study indicated that NLK may have a crucial role in promoting the aggressive phenotypes of CRC and therefore may have the potential for use as a prognostic marker of CRC.

\section{Acknowledgements}

The present study was supported by a grant from the Natural Science Foundation of Shandong Province (no.ZR2011HQ054).

\section{References}

1. Jemal A, Siegel R, Xu J and Ward E: Cancer statistics, 2010. CA Cancer J Clin 60: 277-300, 2010.

2. Gaedcke J, Grade M, Camps J, Søkilde R, Kaczkowski B, Schetter AJ, Difilippantonio MJ, Harris CC, Ghadimi BM, Møller S, et al: The rectal cancer microRNAome - microRNA expression in rectal cancer and matched normal mucosa. Clin Cancer Res 18: 4919-4930, 2012.

3. Siegel R, Ward E, Brawley O and Jemal A: Cancer statistics, 2011: the impact of eliminating socioeconomic and racial disparities on premature cancer deaths. CA Cancer J Clin 61: 212-236, 2011.

4. Zhang S, Cui Y, Weng Z, Gong X, Chen M and Zhong B: Changes on the disease pattern of primary colorectal cancers in Southern China: a retrospective study of 20 years. Int J Colorectal Dis 24: 943-949, 2009.

5. Siegel R, Naishadham D and Jemal A: Cancer statistics. CA Cancer J Clin 63: 11-30, 2013.

6. Speetjens FM, Zeestraten EC, Kuppen PJ, Melief CJ and van der Burg SH: Colorectal cancer vaccines in clinical trials. Expert Rev Vaccines 10: 899-921, 2011.

7. Diep CB, Thorstensen L, Meling GI, Skovlund E, Rognum TO and Lothe RA: Genetic tumor markers with prognostic impact in Dukes' stages B and C colorectal cancer patients. J Clin Oncol 21: 820-829, 2003.

8. Walther A, Johnstone E, Swanton C, Midgley R, Tomlinson I and Kerr D: Genetic prognostic and predictive markers in colorectal cancer. Nat Rev Cancer 9: 489-499, 2009.

9. Roth AD, Tejpar S, Delorenzi M, Yan P, Fiocca R, Klingbiel D, Dietrich D, Biesmans B, Bodoky G, Barone C, et al: Prognostic role of KRAS and BRAF in stage II and III resected colon cancer: results of the translational study on the PETACC-3, EORTC 40993, SAKK 60-00 trial. J Clin Oncol 28: 466-474, 2010.

10. Rajagopalan H, Bardelli A, Lengauer C, Kinzler KW, Vogelstein B and Velculescu VE: Tumorigenesis: RAF/RAS oncogenes and mismatch-repair status. Nature 418: 934, 2002.

11. French AJ, Sargent DJ, Burgart LJ, Foster NR, Kabat BF, Goldberg R, Shepherd L, Windschitl HE and Thibodeau SN: Prognostic significance of defective mismatch repair and BRAF V600E in patients with colon cancer. Clin Cancer Res 14: 3408-3415, 2008.

12. Anastas JN and Moon RT: WNT signalling pathways as therapeutic targets in cancer. Nat Rev Cancer 13: 11-26, 2013. 
13. Ishikawa T, Shimizu D, Kito A, Ota I, Sasaki T, Tanabe M, Yamada A, Arioka H, Shimizu S, Wakasugi J, et al: Breast cancer manifested by hematologic disorders. J Thorac Dis 4 650-654, 2012.

14. Emami KH, Brown LG, Pitts TE, Sun X, Vessella RL and Corey E: Nemo-like kinase induces apoptosis and inhibits androgen receptor signaling in prostate cancer cells. Prostate 69: 1481-1492, 2009.

15. Jung KH, Kim JK, Noh JH, Eun JW, Bae HJ, Xie HJ, Ahn YM, Park WS, Lee JY and Nam SW: Targeted disruption of Nemo-like kinase inhibits tumor cell growth by simultaneous suppression of cyclin D1 and CDK2 in human hepatocellular carcinoma. J Cell Biochem 110: 687-696, 2010

16. Cui G, Li Z, Shao B, Zhao L, Zhou Y, Lu T, Wang J, Shi X, Wang J, Zuo G, et al: Clinical and biological significance of nemo-like kinase expression in glioma. J Clin Neurosci 18: 271-275, 2011

17. Wang S, Wu X, Chen Y, Zhang J, Ding J, Zhou Y, He S, Tan Y, Qiang F, Bai J, et al: Prognostic and predictive role of JWA and XRCC1 expressions in gastric cancer. Clin Cancer Res 18 2987-2996, 2012
18. Weichert W, Röske A, Gekeler V, Beckers T, Ebert MP, Pross M, Dietel M, Denkert C and Röcken C: Association of patterns of class I histone deacetylase expression with patient prognosis in gastric cancer: a retrospective analysis. Lancet Oncol 9: 139-148, 2008.

19. Ishitani $\mathrm{T}$ and Ishitani S: Nemo-like kinase, a multifaceted cell signaling regulator. Cell Signal 25: 190-197, 2013.

20. Ota S, Ishitani S, Shimizu N, Matsumoto K, Itoh M and Ishitani T: NLK positively regulates Wnt/ $\beta$-catenin signalling by phosphorylating LEF1 in neural progenitor cells. EMBO J 31: 1904-1915, 2012.

21. Ishitani T: Context-dependent dual and opposite roles of nemo-like kinase in the Wnt/ $\beta$-catenin signaling. Cell Cycle 11: 1743-1745, 2012.

22. Yasuda J, Yokoo H, Yamada T, Kitabayashi I, Sekiya T and Ichikawa $\mathrm{H}$ : Nemo-like kinase suppresses a wide range of transcription factors, including nuclear factor- $\kappa$ B. Cancer Sci 95: 52-57, 2004.

23. Li M, Zhang S, Wang Z, Zhang B, Wu X, Weng H, Ding Q, Tan Z, Zhang N, Mu J, et al: Prognostic significance of nemo-like kinase (NLK) expression in patients with gallbladder cancer. Tumour Biol 34: 3995-4000, 2013. 